\title{
Transfer Reactions on Neutron-rich Nuclei at REX-ISOLDE
}

Th. Kröll ${ }^{*, \dagger}$, V. Bildstein ${ }^{\dagger}$, K. Wimmer ${ }^{\dagger}$, R. Krücken ${ }^{\dagger}$, R. Gernhäuser ${ }^{\dagger}$, R. Lutter**, W. Schwerdtfeger**, P. Thirolf**, B. Bastin ${ }^{\ddagger}$, N. Bree ${ }^{\ddagger}$, J. Diriken ${ }^{\ddagger}$, M. Huyse ${ }^{\ddagger}$, N. Patronis ${ }^{\ddagger}$, R. Raabe $e^{\ddagger}$, P. Van Duppen ${ }^{\ddagger}$, P. Vermaelen ${ }^{\ddagger}$, J. Cederkäll ${ }^{\S}$, E. Clément ${ }^{\S}$, J. Van de Walle ${ }^{\S}$, D. Voulot ${ }^{\S}$, F. Wenander ${ }^{\S}$, A. Blazhev ${ }^{\mathbb{I}}$, M. Kalkühler ${ }^{\text {II }}$, P. Reiter ${ }^{\text {II }}$, M. Seidlitz ${ }^{\mathbb{T}}$, N. Warr ${ }^{\mathbb{I}}$, A. Deacon $\|$, C. Fitzpatrick" , S. Freeman $\|$, S. Das Gupta ${ }^{\dagger \dagger}$, G. Lo Bianco ${ }^{\dagger \dagger}$, S. Nardellit ${ }^{\dagger \dagger}$, E. Fiori ${ }^{\ddagger \ddagger}$, G. Georgiev ${ }^{\ddagger \ddagger}$, M. Scheck ${ }^{\|}$, L. M. Fraile ${ }^{\S \S}$, D. Balabanski ${ }^{* I T I}$, T. Nilsson ${ }^{* * *}$, E. Tengborn ${ }^{* * *}$, J. Butterworth" , B. S. Nara Singh", L. Angus ${ }^{t \dagger}$, R. Chapman ${ }^{\dagger \dagger}$, B. Hadinia ${ }^{\dagger \dagger}$, R. Orlandi ${ }^{\dagger \dagger \dagger}$, J. F. Smith ${ }^{\dagger \dagger \dagger}$, P. Wady ${ }^{\dagger \dagger \dagger}$, G. Schrieder*, M. Labiche ${ }^{\ddagger}$, J. Johansen ${ }^{\S \S \S}$, K. Riisager ${ }^{\S \S}$, H. B. Jeppesen ${ }^{\text {IIIIII }}$, A. O. Macchiavelli ${ }^{\text {IIIIII }}$, T. Davinson ${ }^{\dagger \dagger \dagger}$ and the REX-ISOLDE and MINIBALL collaborations ${ }^{\S}$

${ }^{*}$ Institut für Kernphysik, Technische Universität Darmstadt, Germany ${ }^{\dagger}$ Physik-Department E12, Technische Universität München, Garching, Germany

${ }^{* *}$ Fakultät für Physik, Ludwig-Maximilians-Universität München, Garching, Germany

${ }^{\ddagger}$ Instituut voor Kern- en Stralingsfysica, Katholieke Universiteit Leuven, Belgium ${ }^{8}$ CERN, Genève, Switzerland

II Institut für Kernphysik, Universität zu Köln, Germany

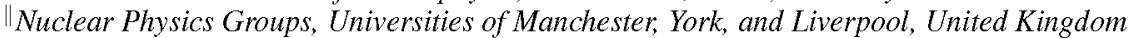

${ }^{\dagger \dagger}$ INFN and Dipartimento di Fisica, Università di Camerino, Italy

${ }^{\ddagger}$ Centre de Spectrométrie Nucléaire et de Spectrométrie de Masse, Orsay, France

${ }^{\S \S}$ Facultad de Sciencias Físicas, Universidad Complutense, Madrid, Spain

IIIINRNE, Bulgarian Acadamy of Sciences, Sofia, Bulgaria

${ }^{*+*}$ Fundamental Fysik, Chalmers Tekniska Högskola, Göteborg, Sweden

${ }^{\dagger}$ Nuclear Physics Groups, Universities of the West of Scotland and Edinburgh, United Kingdom

玤Daresbury Laboratory, Warrington, United Kingdom

${ }^{8 \S}$ Institut for Fy sik og Astronomi, Ârhus Universitet, Denmark

IIII Lawrence Berkeley National Laboratory, USA

\begin{abstract}
We report on one- and two-neutron transfer reactions to study the single-particle properties of nuclei at the border of the "island of inversion". The (d,p)- and $(t, p)$-reactions in inverse kinematics on the neutron-rich isotope ${ }^{30} \mathrm{Mg}$, delivered as radioactive beam by the REX-ISOLDE facility, have been investigated. The outgoing protons have been detected and identified by a newly built array of Si detectors. The $\gamma$-decay of excited states has been detected in coincidence by the MINIBALL array. First results for ${ }^{31} \mathrm{Mg}$ and from the search for the second, spherical, $0^{+}$state in ${ }^{32} \mathrm{Mg}$ are presented.
\end{abstract}

Keywords: transfer reaction, gamma-ray spectroscopy, neutron-rich nuclei

PACS: $25.60 . \mathrm{Je}, 23.20 . \mathrm{g}, 27.30 .+\mathrm{t}$

CP1165, Nuclear Structure and Dynamics '09

edited by M. Milin, T. Nikšić, D. Vretenar, and S. Szilner

(C) 2009 American Institute of Physics 978-0-7354-0702-2/09/\$25.00 


\section{MOTIVATION}

Light-ion induced transfer reactions, like $(\mathrm{d}, \mathrm{p})$ or $(\mathrm{t}, \mathrm{p})$, offering selectivity to both kinematical matching conditions and nuclear structure are a well-established spectroscopic tool to study single-particle properties of nuclear states. For radioactive ions, the reactions have to performed in inverse kinematics. From the energies of the outgoing particles the (single-particle) level energies can be determined. Their angular distributions lead to spin and parity assignments. Both are assisted by the observation of coincident $\gamma$-rays. Eventually, the (relative) spectroscopic factors deduced from the cross sections allow to extract information on the configurations forming the populated state.

However, there are conceptual limitations. Transfer reactions probe only the wave functions on the nuclear surface. The analysis is based on phenomenological approaches (optical model, DWBA) and, therefore, less well under control compared e.g. to Coulomb excitation. Finally, the observed quenching of spectroscopic factors is an open problem but on the other hand also an opportunity to probe the validity of single-particle models. Despite these facts, transfer reactions have been a successful tool in nuclear spectroscopy of stable nuclei for many decades.

Concerning the study of radioactive nuclei, three points are worth to be mentioned. As population mechanism for neutron-rich nuclei a neutron-transfer reaction starting from a less neutron-rich nucleus may be competitive to a direct production in certain cases. Due to its selectivity, states may be populated which are not accessible by other methods, like Coulomb excitation, $\beta$-decay, or fragmentation. In particular, the pair transfer is a valuable tool to study phenomena like shape coexistence and pairing correlations.

In practice, there are also challenges and (open) questions. The parameters of the optical potentials are not known and have to extracted from elastic scattering or extrapolated from stable systems. At REX-ISOLDE, the beam energy is limited to $3 \mathrm{MeV} / \mathrm{u}$. This, together with the low $Q$ values for neutron transfer on neutron-rich nuclei, causes low energies of the outgoing protons, smooth angular distributions, and possible inconsistencies in the determination of spectroscopic factors. These points have been investigated in detail for a stable system [1]. Despite the low beam energies, the observed protons still originate from direct reactions rather than from fusion-evaporation reactions because thermal energy spectra and isotropic angular distributions have not been observed experimentally. As statistical process, only neutrons will be evaporated because their separation energy in neutron-rich nuclei is much smaller compared to that of protons. This simple argument has been confirmed by Hauser-Feshbach calculations [2].

\section{EXPERIMENTAL SET-UP}

ISOLDE is the radioactive beam facility at CERN having more than 40 years of experience in producing RIBs by a $1.4 \mathrm{GeV}$ proton beam impinging on a solid target, in our case $\mathrm{UC}_{x}$. The exotic species are extracted as singly-charged ions and mass separated. In REX-ISOLDE, this low-energy beam is cooled and bunched in a penning trap, charge bread to $A / q \approx 4$ in an EBIS, and finally post-accelerated by a linac to energies up to $3 \mathrm{MeV} / \mathrm{u}$. REX-ISOLDE has delivered since 2002 more than 60 RIBs to experiments.

The experiments reported in this contribution require the coincident measurement of 

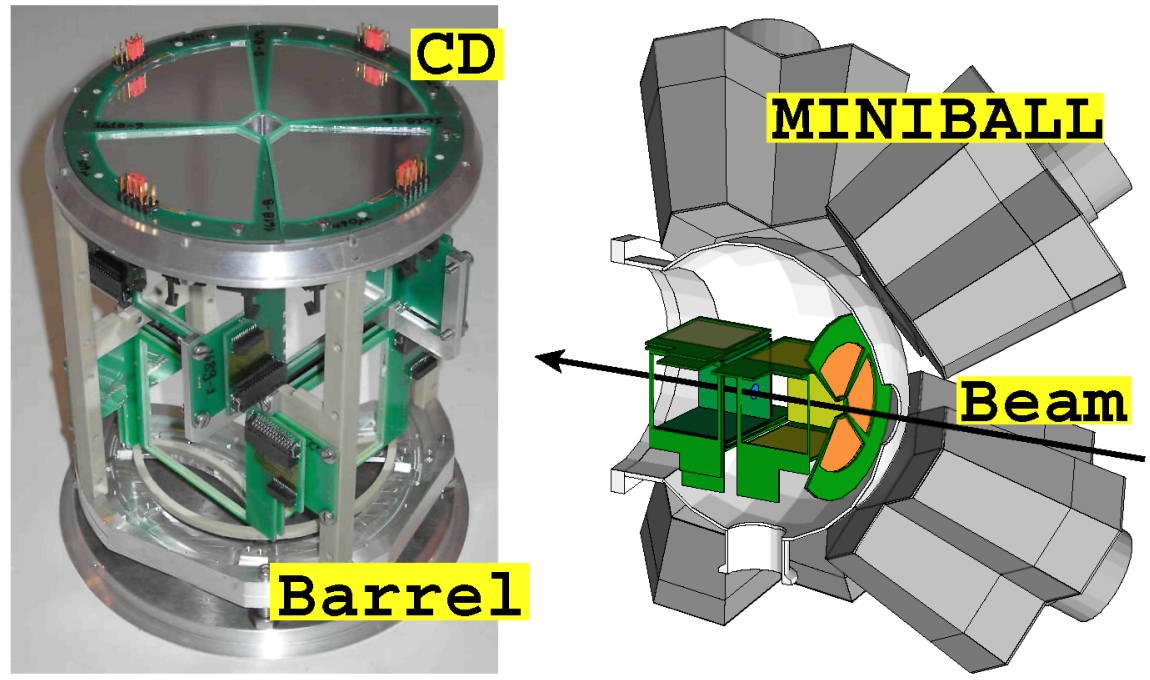

FIGURE 1. Photo (left) and drawing mounted inside of MINIBALL (right) of the new Si array.

the light target-like particles and the $\gamma$-rays. The latter are detected by the MINIBALL array consisting of 24 6-fold segmented HPGe detectors [3]. The photopeak efficiency is around $3 \%$ at $1333 \mathrm{keV}$ with the particle detector inside. This Si detector array has been newly built and offers a large solid angle, position sensitivity, and the capability to identify light particles by the $\Delta E-E$ method. The array consists of a DSSSD detector $(500 \mu \mathrm{m})$, so-called CD detector, in backward direction and a barrel formed by 8 quadratic detectors: stacks of $140 \mu \mathrm{m}$ and $1000 \mu \mathrm{m}$ detectors in forward direction or a single $500 \mu \mathrm{m}$ detector in backward direction [4] (Fig. 1, left). The $140 \mu \mathrm{m}$ and $500 \mu \mathrm{m}$ detectors are read out via resistive strips. The forward DSSSD detector is still missing. The particle identification seemed to be important only in forward direction as backwards only protons originating from transfer reactions are expected. However, it turned out that electrons from the $\beta$-decay of scattered beam particles are badly separated from the low-energy protons. Therefore, the first version of the set-up built and used in 2007 has been upgraded in 2008 by using in forward and backward direction for the barrel the same type of detector stacks and by backing the CD detector with an unsegmented Si detector. As all light ions in backward direction are stopped in the first Si layer, the second only serves to discriminate electrons. The drawing in Fig. 1 (right) shows the arrangement of the Si array inside of MINIBALL as it has been implemented into GEANT4 [5] for simulation.

Additionally, a segmented diamond detector on the target ladder which can be moved at target position and an active collimator with four PIN diodes in front of the chamber for beam focusing have been included.

This set-up was a major upgrade of the instrumentation available at REX-ISOLDE and has been funded by TU München, KU Leuven, University of Edinburgh, and CSNSM Orsay. For 2009, the upgrade to the final set-up is planned. 


\section{ISLAND OF INVERSION}

Although it has been discovered already more than 30 years ago, the structure of nuclei within the "island of inversion" [6,7], a region of nuclei with deformed ground states in the sea of spherical $s d$-nuclei, is still not fully understood. The gross features of this inversion are interpreted as effect of a residual interaction derived from the tensor part of the nuclear force $[8,9]$. The intruder $f p$-orbitals are lowered with respect to the normal $s d$-orbitals and excitations of neutrons to the $f p$-orbitals are energetically favoured. This breaking of the shell closure at $N=20$ leads to deformed states.

At the north-west coast of the island, the even $\mathrm{Mg}$ isotopes have been investigated by lifetime measurements at ISOLDE [10] and by Coulomb excitation at "safe" energies at REX-ISOLDE [11, 12] as well as at intermediate energies, e.g. Refs. [13, 14]. The results establish consistently a nearly spherical shape of the ground state and the first $2^{+}$state in ${ }^{30} \mathrm{Mg}$ but deformed shapes for the corresponding states in ${ }^{32} \mathrm{Mg}$. Hence, the former is outside whereas the latter is inside of the "island of inversion". This interpretation is supported by theory. A good agreement has been achieved by both modern large scale shell model calculations, e.g. Refs. [15, 16], and a "beyond mean field" approach [17]. Therefore, the underlying physics seems to be well understood.

It was a surprise that in an experiment at ISOLDE for the ground state of ${ }^{31} \mathrm{Mg}$, the nucleus right on the shore of the island, a $1 / 2^{+}$assignment was found $[18,19]$ which is in contrast to all theoretical predictions. A possible solution was an adjustment of a few matrix elements of the monopole interaction used in the shell model calculations [20]. In this approach, the ground state and the first excited state in ${ }^{31} \mathrm{Mg}$ are largely dominated by $2 \mathrm{p}-2 \mathrm{~h}$ intruder configurations ( $93 \%$ and $95 \%$ of the wave function, respectively) with a $f p$-shell occupancy close to 2 . Hence, the transition into the island is much steeper than e.g. in Na. However, there is no consistent microscopic description of this region.

Aiming to study the single-particle properties also of excited states in ${ }^{31} \mathrm{Mg}$, we investigated the $\mathrm{d}\left({ }^{30} \mathrm{Mg},{ }^{31} \mathrm{Mg}\right)$ p reaction. $\mathrm{A}^{30} \mathrm{Mg}$ at $2.86 \mathrm{MeV} / \mathrm{u}$ with a, due to technical problems, low intensity of $10^{4}$ part $/ \mathrm{s}$ impinged on a $1 \mathrm{mg} / \mathrm{cm}^{2}$ deuterated PE target. The experiment has been performed in 2007 and the analysis is still in progress [21].

In Fig. 2 (left), the $\Delta E-E$ particle identification plot is shown. Clearly, the different particles, i.e. p, $d, t$, and, $\beta$, are separated. As the energy resolution is not sufficient to identify the populated states, the $\gamma$-rays are needed. Fig. 2 (right) shows part of the $\gamma$-ray spectrum in coincidence with the protons. Gating on the $170 \mathrm{keV}$ transition, the angular distribution of the respective protons is consistent with a $\Delta \ell=1$ orbital momentum transfer, giving support for the tentative $3 / 2^{-}$assignment for the state at $221 \mathrm{keV}$.

An alternative look into the evolution of the single-particle structure can be obtained from the study of the excited $0^{+}$states. The configuration which forms the ground state in ${ }^{30} \mathrm{Mg}$ becomes an excited $0^{+}$state in ${ }^{32} \mathrm{Mg}$ and vice versa. The coexistence of $0^{+}$ states with different shapes and the inversion of their order is a direct consequence of the migration of the single-particle levels.

In ${ }^{30} \mathrm{Mg}$, the second $0^{+}$state at $1789 \mathrm{keV}$ is quite well known as it is populated in the $\beta$-decay of ${ }^{30} \mathrm{Na}$. Its $\gamma$-decay to the $2^{+}$state and its lifetime [10] as well as its E0 decay to the ground state [22] have been measured at ISOLDE. The results are consistent with a deformed shape and a small mixing with the ground state [22].

The excited $0^{+}$state in ${ }^{32} \mathrm{Mg}$ has not been observed experimentally so far. It is not 

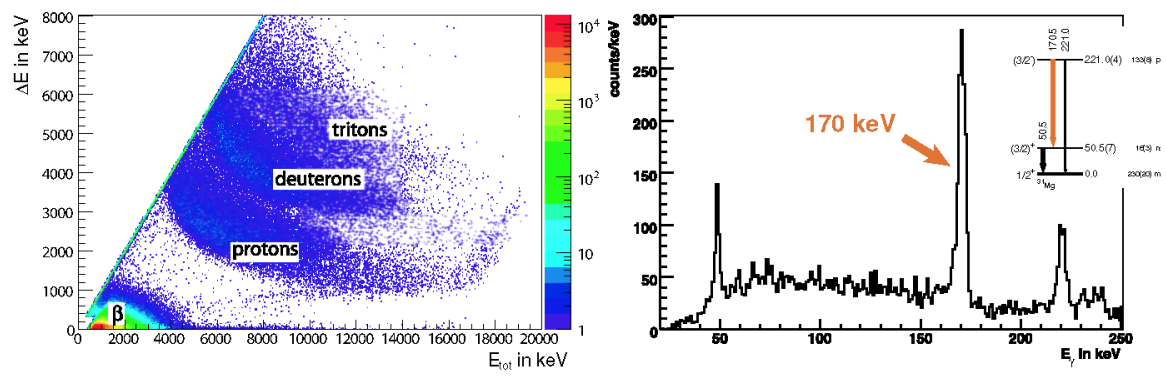

FIGURE 2. PID plot (left) and part of the $\gamma$-ray spectrum in coincidence with the protons (right).

populated in the $\beta$-decay of ${ }^{32} \mathrm{Na}[10,23]$, in Coulomb excitation, or in fragmentation reactions [24]. The theoretical predictions range from $1.4 \mathrm{MeV}$ to $3 \mathrm{MeV}[25,26]$.

As its particle-hole structure is assumed to be similar to that of the ground state of ${ }^{30} \mathrm{Mg}$, hence a spherical $0 \mathrm{p}-0 \mathrm{~h}$ configuration, it should be selectively populated in a two-neutron transfer reaction because an large overlap of the wave functions means a large spectroscopic factor. The population of other states in ${ }^{32} \mathrm{Mg}$ with $2 \mathrm{p}-2 \mathrm{~h}$ intruder configuration, e.g. the first $2^{+}$state, should be disfavoured.

In order to search for the second $0^{+}$state in ${ }^{32} \mathrm{Mg}$, the $\mathrm{t}\left({ }^{30} \mathrm{Mg},{ }^{32} \mathrm{Mg}\right) \mathrm{p}$ reaction has been employed. $\mathrm{A}^{30} \mathrm{Mg}$ beam at $1.83 \mathrm{MeV} / \mathrm{u}$ with an intensity of $4 \cdot 10^{4} \mathrm{part} / \mathrm{s}$ impinged on a $0.5 \mathrm{mg} / \mathrm{cm}^{2}$ Ti target loaded with tritium $\left({ }^{3} \mathrm{H} / \mathrm{Ti}=1.5\right)$. The experiment has been performed in 2008 and the analysis is in progress [27].

From the first preliminary results several interesting points can be mentioned. Protons from the transfer reaction are seen, kinematically clearly separated from elastically scattered protons (and tritons). Hence, the two-neutron transfer works. No deuterons are observed, as the $Q=-3.9 \mathrm{MeV}$ disfavours strongly the $(\mathrm{t}, \mathrm{d})$ reaction because of kinematic mismatching. There is also no evidence for the population of the $2^{+}$state, consistent with the different structure and the negative $Q=-1.2 \mathrm{MeV}$. Reconstructing the excitation energies from the proton energies, the population of two states can be identified: the ground state and a new state at around $1.1 \mathrm{MeV}$. The first can be understood by the small $Q=-0.3 \mathrm{MeV}$, near to the optimal value of $Q=0$, and the small configuration mixing in the wave functions. The angular distributions of the respective protons indicate a $\Delta \ell=0$ orbital momentum transfer for both states. Therefore, the new state is a promising candidate for the long-searched second $0^{+}$state in ${ }^{32} \mathrm{Mg}$.

\section{Summary and outlook}

Further experiments using the described set-up have already been approved: $\mathrm{d}\left({ }^{11} \mathrm{Be}\right.$,

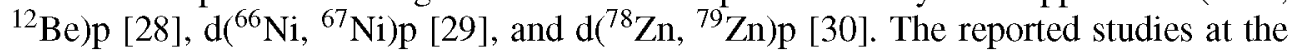
shore of the "island of inversion" will be extended to neighbouring $\mathrm{Al}$ or Na isotopes.

For the farer future experiments addressing many interesting topics like the quenching of spectroscopic factors, shape coexistence in the region of neutron-deficient $\mathrm{Pb}$ isotopes, paring correlations in exotic nuclei (surface pairing, $T=0 \mathrm{np}$-paring in $N=Z$ 
nuclei) etc. are already under discussion.

Most of these experiments will profit from or even require the upgrade of REXISOLDE to HIE-ISOLDE [31]. Apart from a step-wise increase in energy to $5.5 \mathrm{MeV} / \mathrm{u}$ and later to $10 \mathrm{MeV} / \mathrm{u}$, larger intensities and better beam qualities are envisioned. Studies for a $0^{\circ}$ separator to identify also the heavy transfer products have been started.

We conclude that transfer reactions are valuable tool for nuclear spectroscopy and will considerably contribute to the understanding of exotic nuclei.

\section{ACKNOWLEDGMENTS}

This work is supported by the German BMBF under grant No. 06MT238, by the MLL, by the EU through EURONS (contract No. 506065), by the state of Hessen within the HIC for FAIR programme and the LOEWE excellence initiative, and by the DFG cluster of excellence Universe (www.universe-cluster.de). REX-ISOLDE and MINIBALL are supported by the German BMBF, the Belgian FWO-Vlaanderen and IAP, the U.K. EPRSC, and the EU, as well as the ISOLDE collaboration.

\section{REFERENCES}

1. M. Mahgoub, $\mathrm{PhD}$ thesis (TU München, 2008).

2. M. Pantea, $\mathrm{PhD}$ thesis (TU Darmstadt, 2005).

3. J. Eberth, et al., Prog. Part. Nucl. Phys. 46, 389 (2001).

4. V. Bildstein, et al., Prog. Part. Nucl. Phys. 59, 386 (2007).

5. GEANT4, http://geant4.web.cern.ch/.

6. C. Thibault, et al., Phys. Rev. C 12, 644 (1975).

7. E. K. Warburton, et al., Phys. Rev. C 41, 1147 (1990).

8. T. Otsuka, et al., Eur. Phys. J. A 15, 151 (2002).

9. T. Otsuka, et al., Phys. Rev. Lett. 95, 232502 (2005).

10. H. Mach, et al., Eur. Phys. J. A 25, 105 (2005).

11. O. Niedermaier, et al., Phys. Rev. Lett. 94, 172501 (2005).

12. O. Niedermaier, $\mathrm{PhD}$ thesis (Universität Heidelberg, 2005); to be published.

13. T. Motobayashi, et al., Phys. Lett. 346B, 9 (1995).

14. J. A. Church, et al., Phys. Rev. C 72, 054320 (2005).

15. Y. Utsuno, et al., Phys. Rev. C 60, 054315 (1999).

16. E. Caurier, et al., Nucl. Phys. A 693, 374 (2001).

17. R. Rodríguez-Guzmán, et al., Nucl. Phys. A 709, 201 (2002).

18. G. Neyens, et al., Phys. Rev. Lett. 94, 022501 (2005).

19. M. Kowalska, PhD thesis (Universität Mainz, 2006).

20. F. Maréchal, et al., Phys. Rev. C 72, 044314 (2005).

21. V. Bildstein, $\mathrm{PhD}$ thesis (TU München, 2009).

22. W. Schwerdtfeger, et al., Phys. Rev. Lett. (in press).

23. C. M. Mattoon, et al., Phys. Rev. C 75, 017302 (2007).

24. M. Gelin, $\mathrm{PhD}$ thesis (Université de Caen, 2007).

25. D. Guillemaud-Mueller, et al., Eur. Phys. J. A 13, 63 (2002).

26. T. Otsuka, et al., Eur. Phys. J. A 20, 69 (2004).

27. K. Wimmer, $\mathrm{PhD}$ thesis (TU München, expected for 2010).

28. J. Johansen, et al., contribution to this conference.

29. N. Patronis, et al., Proposal CERN-INTC-2008-007, INTC-P-238 (2008).

30. R. Orlandi, et al., Proposal CERN-INTC-2009-017, INTC-P-264 (2009).

31. HIE-ISOLDE - the scientific opportunities, Eds. K. Riisager, P. Butler, R. Krücken, CERN-2007-008. 